\title{
Carbohydrate Composition of Endotoxins from $R$-type Isogenic Mutants of Shigella sonnei Studied by Capillary Electrophoresis and GC-MS ${ }^{\dagger}$
}

\author{
Annamária Bui, ${ }^{a}$ Anikó Kilár, ${ }^{\text {b,c }}$ Ágnes Dörnyei, ${ }^{\text {a,c }}$ Viktória Poór, ${ }^{a}$ \\ Krisztina Kovács, ${ }^{\mathrm{b}}$ Béla Kocsis, ${ }^{\mathrm{b}}$ and Ferenc Kilár ${ }^{\mathrm{a}, \mathrm{c}, *}$ \\ anstitute of Bioanalysis, Faculty of Medicine, University of Pécs, Szigeti út 12., H-7624 Pécs \\ ${ }^{\mathrm{b}}$ Department of Medical Microbiology and Immunology, Faculty of Medicine, \\ University of Pécs, Szigeti út 12., H-7624 Pécs, Hungary \\ ${ }^{c}$ Department of Analytical and Environmental Chemistry, Faculty of Sciences, \\ University of Pécs, Ifjúság útja 6., H-7624 Pécs, Hungary
}

RECEIVED NOVEMBER 9, 2010; REVISED FEBRUARY 5, 2011; ACCEPTED MAY 13, 2011

\begin{abstract}
The carbohydrate composition of the rough-type endotoxins (lipopolysaccharides, LPSs) from Shigella sonnei mutant strains (Shigella sonnei phase II - 4303, 562H, R41 and 4350) was investigated by capillary electrophoresis and GC-MS. The monosaccharides obtained by hydrolysis were determined by capillary electrophoresis combined with laser induced fluorescence detection (CE-LIF) after labeling with 8-aminopyrene-1,3,6-trisulfonic acid (APTS) and by GC-MS as alditol-acetate derivatives. It was obtained that the lipopolysaccharides of the isogenic rough mutants are formed in a step-like manner, containing no heptose (4350), one D-glycero-D-mannoheptose $(562 \mathrm{H})$, or two or three L-glycero-Dmannoheptoses $(R 41,4303$, respectively) in the deep core region. Besides the heptoses, the longest LPS from the mutant Shigella sonnei 4303 contains hexoses, such as glucoses and galactoses, in a proportion of approximately $3: 2$. This study provides a comprehensive comparison of the variability in the carbohydrate part of the rough endotoxins extracted from Shigella sonnei isogenic mutants. (doi: $10.5562 / \mathrm{cca} 1795$ )
\end{abstract}

Keywords: lipopolysaccharide structure, Shigella sonnei rough mutant, D-glycero-D-mannoheptose, capillary electrophoresis, CE-LIF, GC-MS

\section{INTRODUCTION}

The structural composition of the bacterial endotoxins (also known as lipopolysaccharides or LPSs) is of importance due to their pathophysiological impact. The endotoxins are cell wall components of the Gramnegative smooth $(S)$ or rough $(R)$ bacteria, ${ }^{1}$ and consist of two or three main parts: (i) a glycolipid region, called lipid A, (ii) an oligosaccharide (OS) region, called core, to which (iii) a polysaccharide region, called O-chain, is attached in the $S$-type endotoxins. ${ }^{2}$ Spontaneous or induced mutations of $S$-type strains may cause loss of carbohydrate constituents (O-chains and part of the core) in the endotoxins, resulting in the $R$-type lipopolysaccharides. ${ }^{3}$ There is a high degree in the variability of the polysaccharide chains (O-chains) amongst the different bacteria, while the core oligosaccharides have a more conservative structure. The oligosaccharide core is formed by several sugar units such as 3-deoxyD-manno-2-octulosonic acid (Kdo), heptose(s) (Hep, i.e., L-glycero-D-mannoheptose or D-glycero-D-mannoheptose) and neutral hexoses like D-glucose (D-Glc), D-galactose (D-Gal) or their derivatives. One (or more) Kdo residue(s) is (are) found in the connection part between the core OS and the lipid A. The lipid A usually consists of a D-glucosamine (GlcN) disaccharide backbone acylated with $\beta$-hydroxylated fatty acids.

The significance of the endotoxins in living organisms is due to the antigenicity of the O-antigen as well as the toxic character of the lipid A moiety ${ }^{4}$. The innermost region of an endotoxin, consisting of the lipid A and the Kdo residues, is essential for the viability of the Gram-negative bacteria.

Shigella sonnei (S. sonnei) phase $\mathrm{I}^{3}$ is an important member of the Enterobacteriaceae family, and causes dysentery. This $S$-type strain can spontaneously mutate to the non-pathogenic $R$-type $S$. sonnei 4303 (phase II), ${ }^{3}$ which has the same core region but it lacks the O-chain ${ }^{3,5,6}$ due to plasmid-loss. A series of $R$-type isogenic mutants, namely S. sonnei $\mathrm{R} 41,562 \mathrm{H}^{7}$ and $4350^{8}$

\footnotetext{
$\uparrow$ Presented at the $10^{\text {th }}$ International Symposium and Summer School on Bioanalysis within the CEEPUS Network CII-HU-001004-0910, Zagreb, Croatia, July 2010.

* Author to whom correspondence should be addressed. (E-mail: ferenc.kilar@aok.pte.hu)
} 
have been developed by ethyl-methyl-sulfonate induced mutagenesis $^{9}$ from the parent strain, S. sonnei 4303 (being already a mutant). The mutant strains were isolated with the help of $R$-specific phages. Previous studies claim that the $S$. sonnei 4350 strain is a heptosetransferase-less mutant, ${ }^{8}$ while the $\mathrm{S}$. sonnei $562 \mathrm{H}$ strain lacks the ADP-L-glycero-D-mannoheptose-6-epimerase enzyme. ${ }^{7,10}$

Although, the carbohydrate regions of the endotoxins from the $S$-type $S$. sonnei phase I and the $R$-type $S$. sonnei 4303 (phase II) have been studied extensively by different methods, ${ }^{11-15}$ the structures of the LPS extracted from the isogenic deep rough mutants $(562 \mathrm{H}$, $R 41,4350$ ) have not been fully characterized (the molecular masses of these LPSs were determined by mass spectrometry). ${ }^{16}$

In this paper we describe the usefulness of capillary electrophoresis combined with laser induced fluorescence detection (CE-LIF) and GC-MS methods in the identification of the sugar moiety of endotoxins. These methods were used to provide a detailed profiling of the LPSs of isogenic deep rough mutants of $S$. sonnei.

\section{EXPERIMENTAL}

\section{Materials}

All monosaccharide standards (D-ribose, D-mannose, D-glucose, D-galactose, inositol), as well as APTS (8-aminopyrene-1,3,6-trisulfonic acid), were supplied by Sigma-Aldrich (St. Louis, Missouri, USA). Sodium cyanoborohydride $\left(1.0 \mathrm{M} \mathrm{NaBH}{ }_{3} \mathrm{CN}\right.$ solution in tetrahydrofuran) was obtained from Aldrich (St. Louis, Missouri, USA) and citric acid and boric acid were purchased from Reanal (Budapest, Hungary). All other reagents were of analytical grade.

\section{Bacterial Strains}

S. sonnei phase I bacteria were cultured at $37{ }^{\circ} \mathrm{C}$ in a laboratory fermentor in a beef extract peptone broth at $\mathrm{pH}=7.2$, until they reached the late logarithmic phase (about $10 \mathrm{~h}$ ), and then collected by centrifugation. ${ }^{7}$ Spontaneous mutation of the smooth colonies of this strain leads to the rough or $R$-type $S$. sonnei 4303 (phase II) of which further $R$ mutants, named $S$. sonnei $R 41$, $562 \mathrm{H}$ and 4350 , were produced by induced mutation using ethyl-methyl-sulfonate as mutagenic agent. ${ }^{17}$ The mutant bacteria were isolated and classified on the basis of their core-specific phage sensitivity. ${ }^{8}$ The bacterial endotoxins were extracted from acetone-dried organisms by the phenol-chloroform-petroleum ether method $^{18}$ and were lyophilized.

\section{Preparation of Monosaccharides from Endotoxins}

The endotoxins (50-200 mg) were hydrolyzed with $1 \%$ acetic acid ("mild hydrolysis") at $100{ }^{\circ} \mathrm{C}$ for $90 \mathrm{~min}$ to separate the carbohydrate part from the lipid A. The lipid A part of each endotoxin was removed by centrifugation $\left(10000 \times \mathrm{g}, 15 \mathrm{~min}, 4^{\circ} \mathrm{C}\right)$. The supernatants (containing the truncated core parts and other small Kdo fragments, as well as minor amount of intact endotoxin due to incomplete hydrolysis), were freeze-dried, redissolved in $1 \mathrm{ml}$ of pyridine-acetate buffer $(4 \mathrm{ml}$ pyridine, $20 \mathrm{ml}$ acetic acid in $1000 \mathrm{ml}$ deionized water) and fractionated by gel chromatography on a Sephadex G-50 (Sigma-Aldrich, USA) column $(2.5 \times 80 \mathrm{~cm})$ using the same buffer. ${ }^{19}$ Fractions ( $4 \mathrm{ml}$ each) were collected and tested by the phenol-sulfuric acid method ${ }^{20}$ for the carbohydrate content. Following lyophilization, the fraction materials were hydrolyzed in $0.5 \mathrm{M}$ sulfuric acid for $14 \mathrm{~h}$ at $100{ }^{\circ} \mathrm{C}$ in order to obtain the monosaccharide components. Barium hydroxide was used after hydrolysis to remove the sulfate. The hydrolyzed samples, containing the monosaccharides, were then lyophilized.

\section{Thin Layer Chromatography}

Samples, containing the monosaccharide mixture from the endotoxins, were run in a Shandon's chromatography vessel $^{21}$ on a Cellulose DC Plastikfolia (Merck, Darmstadt, Germany) thin layer with buthanol/pyridine/water mixture $(6: 4: 3)$, which is optimal for neutral sugars. An aqueous mixture of D-ribose, D-mannose, D-galactose and D-glucose was used as the standard solution. The samples were detected by silver staining as described by Trevelyan et al. ${ }^{22}$

\section{Gas-Chromatography with MS Detection}

The monosaccharides that were isolated from the endotoxins were analyzed in their alditol-acetate forms by GC-MS. The derivatization procedure was used according to Sawardeker et $a .^{23}$ Briefly, the hydrolyzates were reduced with sodium-borohydride at room temperature for $4 \mathrm{~h}$. The excess borohydride was quenched with acetic acid and then the borate was removed with methanol. The samples containing the reduced sugars were then desiccated. Peracetylation was performed with acetic anhydride at $100{ }^{\circ} \mathrm{C}$ for $12 \mathrm{~h}$. The samples, containing the sugar derivatives, were washed with water, desiccated and resuspended in chloroform. The GC-MS system (Agilent Technologies, Waldbronn, Germany) consisted of a gas chromatograph (6890N) and a mass spectrometer (5975) equipped with a quadrupole mass analyzer. The separation was performed on a DB-225 column (Agilent Technologies, Waldbronn, Germany, length: $30 \mathrm{~m}$; inner diameter: 
$0.25 \mathrm{~mm}$; film thickness: $0.15 \mu \mathrm{m})$. Helium was used as

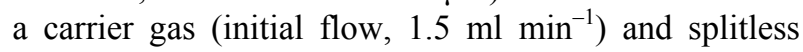
injection was applied. The GC temperature program was as follows: the temperature was raised from the initial $100{ }^{\circ} \mathrm{C}$ to $185^{\circ} \mathrm{C}$ with a rate of $35^{\circ} \mathrm{C} \mathrm{min}{ }^{-1}$. Then, the temperature was raised to $220^{\circ} \mathrm{C}$ at a rate of $5^{\circ} \mathrm{C} \mathrm{min}{ }^{-1}$ and maintained for $60 \mathrm{~min}$. The injector temperature was $250{ }^{\circ} \mathrm{C}$, the transfer line was set at $280{ }^{\circ} \mathrm{C}$, and the ion source was used at $230{ }^{\circ} \mathrm{C}$. The mass spectrometer was operated at $70 \mathrm{eV}$ in the electron impact (EI) mode. The monosaccharides of the samples from the different bacterial strains were identified with the help of the MSlibrary and by spiking the samples with standards. Inositol in its alditol-acetate form was used as internal standard, as none of the samples contains it naturally.

\section{Derivatization of Monosaccharides for CE-LIF Analysis}

$0.1 \mathrm{M}$ monosaccharide solutions were prepared in water, and desiccated in SpeedVac afterward (in $2 \mu \mathrm{l}$ ). The monosaccharides were derivatized ${ }^{24}$ by adding $2 \mu \mathrm{l}$ of $0.1 \mathrm{M}$ APTS in water, $2 \mu \mathrm{l}$ of $0.6 \mathrm{M}$ citric acid and $2 \mu \mathrm{l}$ of $1.0 \mathrm{M}$ sodium cyanoborohydride (in tetrahydrofuran) to the desiccated lyophilisate or crystal powder. The reductive amination was allowed to proceed at $75^{\circ} \mathrm{C}$ for $1 \mathrm{~h}$. The derivatized mixtures were diluted with water to $200 \mu \mathrm{l}$ (or to a higher volume) for proper detection in the CE-LIF experiments.

\section{Capillary Electrophoresis}

$\mathrm{CE}$ analysis of the fluorescently labeled monosaccharides was performed ${ }^{24}$ on a Crystal $300 \mathrm{CE}$ system (Unicam Limited, Cambridge, UK) equipped with a Zetalif laser-induced fluorescence detector (Picometrics, France). Uncoated $20 \mu \mathrm{m}$ ID fused silica capillary with an overall length of $60 \mathrm{~cm}$ and an effective length of 48 $\mathrm{cm}$ (obtained from MicroQuartz, Munich, Germany) was used. Before sample injection the capillary was washed with water for $3 \mathrm{~min}$, then with background electrolyte for $3 \mathrm{~min}$. The sample was injected with 20 or $50 \mathrm{mbar}$ for $0.23 \mathrm{~min}$. The end of the capillary was washed after injection for $0.1 \mathrm{~min}$ by dipping the capillary end in a vial containing buffer to remove the dye attached to the outer surface of the capillary. Experimental conditions were as follows: voltage, $30 \mathrm{kV}$ (resulting current, $21 \mu \mathrm{A}$ ), background electrolyte, 120 $\mathrm{mM}$ borate at $\mathrm{pH}=10.2$. A 50 mbar pressure was applied on the capillary during the electrophoresis to shorten the runtime (the applied pressure did not influence the quality of the separation). A $488 \mathrm{~nm}$ Ar-ion laser (Spectra-Physics Inc., Mountain View, CA, USA) was used for the LIF detection of the APTS-labeled samples. Data were collected by the Axxi-Chrom 717 Chromatography Data Station (Axxiom Chromatogra- phy Inc., Moorpark, CA, USA). After the electrophoretic run the capillary was washed with $0.1 \mathrm{M} \mathrm{NaOH}$ for 4 $\min$. The fluorescent derivatives of the sugars were identified by spiking the samples with derivatized standards.

\section{RESULTS AND DISCUSSION}

The monosaccharide constituents of $R$-type endotoxins from $S$. sonnei isogenic rough mutants $S$. sonnei 4350 , $562 \mathrm{H}, \mathrm{R} 41$ and 4303 , obtained by a gel-chromatographic separation after hydrolysis, were analyzed by thin layer chromatography (TLC), and derivatives of the hydrolytic products were studied by GC-MS and capillary electrophoresis with LIF detection (CE-LIF).

The first gel-chromatography fractions after hydrolysis, which appeared near the void volume of the separation column, were considered to be the complete endotoxin components, which remained after the incomplete hydrolytic treatment. In the case of 4350 only the complete endotoxin was observed, but no hydrolytic products were obtained in the following fractions, and no monosaccharides (such as heptoses and/or hexoses) were detected by thin layer chromatography, or by other separation methods (results not shown).

Different monosaccharides were, however, obtained upon the hydrolysis of the endotoxins from $S$. sonnei $562 H, R 41$ and 4303. The TLC, GC-MS and CELIF analyses detected heptoses in the hydrolytic mixture of each LPS, and also hexose components in the case of 4303.

The heptoses in the LPSs were either D-glycero-Dmannoheptose (D,D-heptose) or L-glycero-D-mannoheptoses (L,D-heptose) components. The differentiation of these heptose isomers was not possible with thin layer chromatography, since they had the same retention time (results are not shown). In the GC-MS analysis, the alditol-acetate derivatives of the heptoses from the LPS of $S$. sonnei $562 H, R 41$ and 4303 , however, were eluted with different relative retention times $(1.44 \pm 0.02$, $1.59 \pm 0.02$ and $1.60 \pm 0.02$, respectively) compared to the standard peracetylated inositol. This indicates that the heptoses occurring in $R 41$ and 4303 are the same, but different from the heptose occurring in $562 \mathrm{H}$. Figure 1 shows the GC-MS chromatogram of the mixture of alditol-acetate derivatives of monosaccharides obtained by hydrolyzing the endotoxin extracted from Shigella sonnei $562 \mathrm{H}$ and 4303 . The sugar components were identified as hexoses or heptoses by their mass spectra.

Figures $2 \mathrm{a}, 2 \mathrm{~b}$ and $2 \mathrm{c}$ show the electropherograms of the hydrolyzates containing the APTS labeled monosaccharides from $S$. sonnei $562 H$, S. sonnei $R 41$ and $S$. sonnei 4303, respectively. The electrophoretic patterns show that two different heptoses occur in these endotoxins. The electropherogram of the mixture of the hydrolyzates from S. sonnei $562 \mathrm{H}$ and S. sonnei 4303 is 


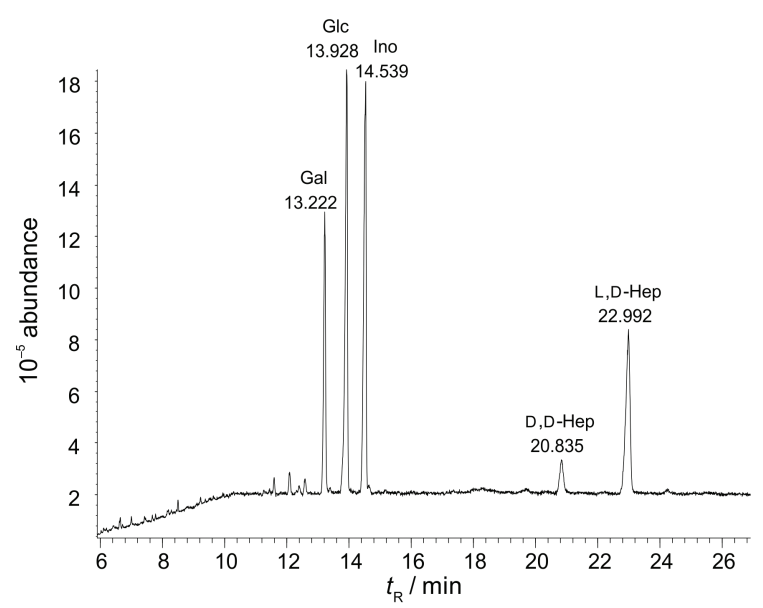

Figure 1. GC-MS chromatogram of the mixture of alditolacetate derivatives of monosaccharides obtained by hydrolyzing the endotoxin from Shigella sonnei $562 \mathrm{H}$ and 4303. The sample was collected by fractionation on a Sephadex G-50 column and then subjected for derivatization. (D,D-Hep: D-glycero-D-mannoheptose, L,D-Hep: L-glycero-D-mannoheptose, Glc: glucose, Gal: galactose, Ino: inositol, used as internal standard). Experimental conditions are described in the Experimental section. presented in Figure 2d. The fluorescent derivatives of the L,D-heptose and D-glucose comigrate during electrophoresis (but based on the peak area ratios, spiking with standard and the GC-MS results, we were able to unequivocally identify the peaks), while the fluorescent derivative of D-galactose migrates with different velocity (Figure 2c). Analyzing the APTS labeled sugar components in the mixture of the hydrolyzates of the two endotoxins from $S$. sonnei $562 H$ and S. sonnei 4303, respectively, two different sugar components, being the D,D- and L,D-heptoses were obtained in the electropherogram (Figure $2 \mathrm{~d}$ ).

Both, the GC-MS and CE-LIF measurements showed the presence of two kinds of hexoses, i.e., D-galactose and D-glucose in the hydrolyzate of the lipopolysaccharide from S. sonnei 4303 (with relative retention times of the alditol-acetate derivatives in GC-MS of 0.91 and 0.96 , respectively). The molar ratio of the D-galactose and D-glucose content estimated from the GC-MS analysis were found to be 2.0:3.3.

The hydrolyzates did not show the presence of any other intact carbohydrate components, neither in the GC-MS, nor in the CE-LIF experiments.
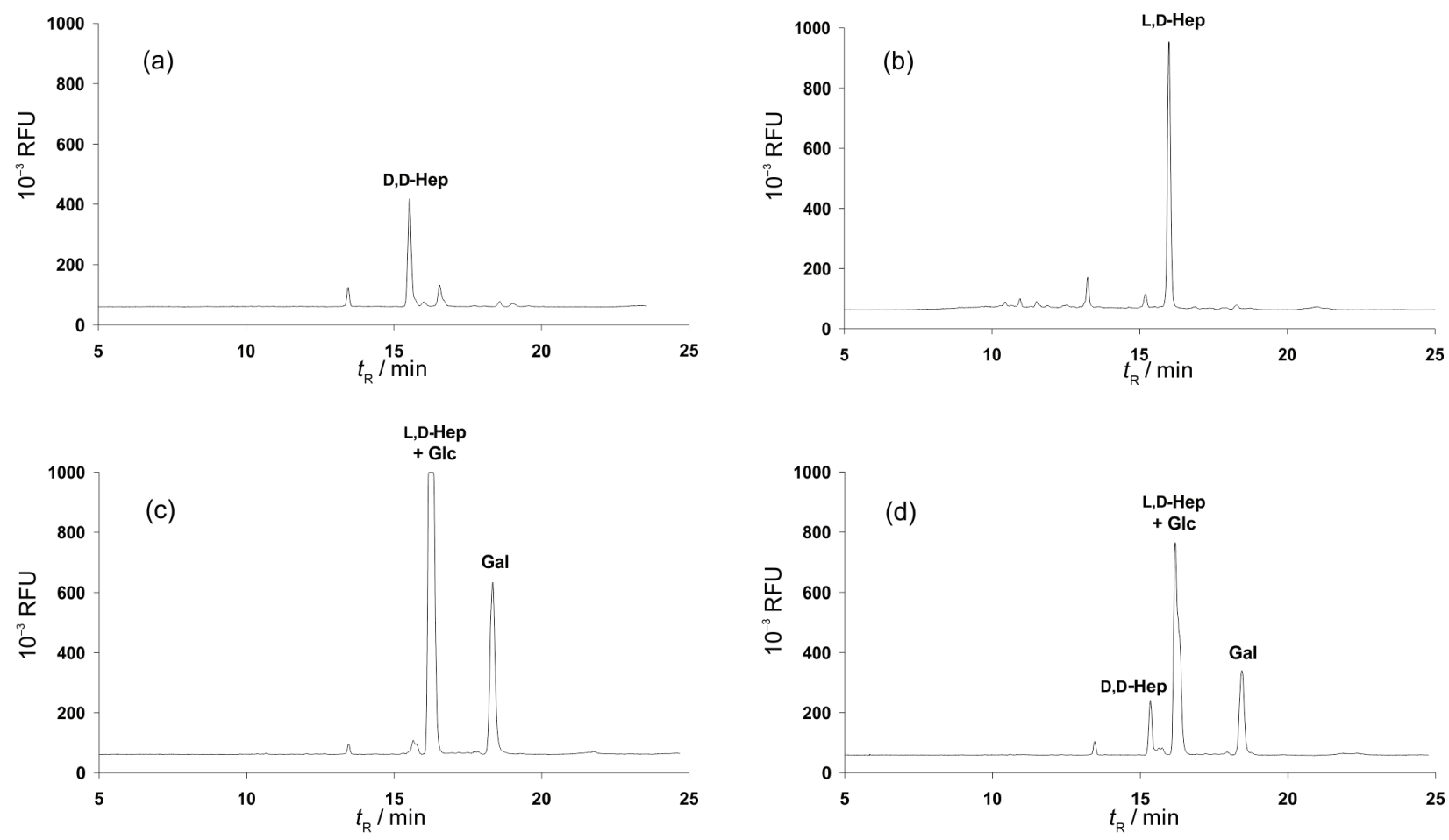

Figure 2. Capillary zone electrophoresis of fluorescently labeled monosaccharides obtained by hydrolyzing the $R$-type endotoxins from $S$. sonnei $562 H$ (a), S. sonnei R41 (b), S. sonnei 4303 (c) and the mixture of S. sonnei $562 H$ and S. sonnei 4303 (d). The samples were collected by fractionation on a Sephadex G-50 column and then subjected for derivatization with APTS. A comigration of the APTS labeled D-glucose and L,D-heptose can be observed (c and d). The two heptose derivatives from $\mathrm{S}$. sonnei $562 \mathrm{H}$ and $S$. sonnei 4303 are separated from each other (d). Experimental conditions are described in the Experimental section. (D,DHep: D-glycero-D-mannoheptose, L,D-Hep: L-glycero-D-mannoheptose, Glc: D-glucose, Gal: D-galactose) 


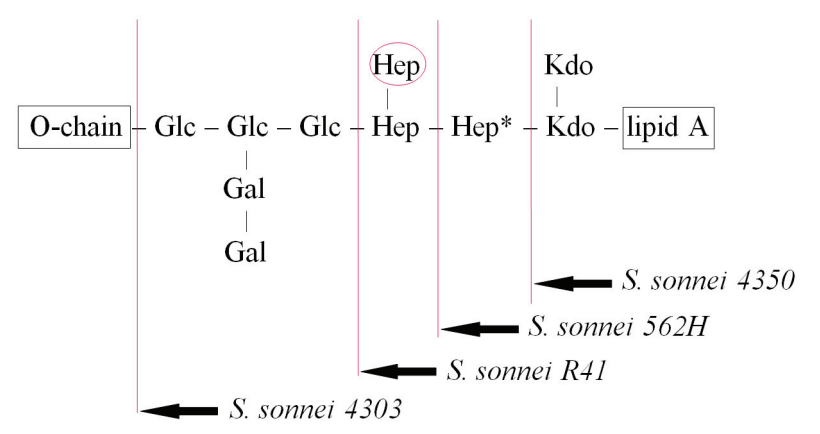

Figure 3. The chemical structures of the $R$-type endotoxins from the isogenic $S$. sonnei mutants, according to the present study and previous investigations. ${ }^{11,14,16}$ The S. sonnei $562 \mathrm{H}$ mutant LPS contains D-glycero-D-mannoheptose $(*)$, while S. sonnei R41 and 4303 LPS contain two or three L-glycero-Dmannoheptoses, respectively (the L,D-heptose in the circle is a constituent only in the endotoxin from $S$. sonnei 4303). The "O-chain" gives a hint to the $S$-type $S$. sonnei phase I endotoxin structure. Kdo: 3-deoxy-D-manno-2-octulosonic acid, Hep: heptose, Glc: D-glucose, Gal: D-galactose.

The sugar composition of the endotoxins from the $S$. sonnei rough mutants show an increasing complexity in the following order, 4350, $562 \mathrm{H}, \mathrm{R} 41$ and 4303 . The recommended structures of the $R$-type lipopolysaccharides are summarized in Figure 3.

In our previous work, ${ }^{8}$ we have reported that S. sonnei 4350 is a heptose-transferase-less mutant, which means that the pathway of the biosynthesis for the heptose unit (the common sugar units in the deep core regions of endotoxins $)^{25}$ is hindered. This strain can produce both, the ADP-D,D-heptose and ADP-L,Dheptose (which are the precursors of the core oligosaccharides of endotoxins), but without the heptosetransferase enzyme it cannot build the heptose(s) into its LPS, thus it is an absolute $R$ mutant, containing no heptose unit in its LPS.

The GC-MS and CE-LIF measurements show that two different heptoses can be found in the three strains. The LPS from $S$. sonnei $562 H$ has one heptose residue in the core, ${ }^{16}$ but according to the GC-MS results (relative retention time values) and the CE-LIF experiments (Figure 2), this heptose differs from the common L,Dheptose constituent, which is a usual constituent in other endotoxin structures. According to the previous assumption of Kontrohr and Kocsis ${ }^{7,10}$ this heptose should be a D,D-heptose, due to the lack of the ADP-L-glycero-Dmannoheptose-6-epimerase enzyme (a general member in LPS biosynthesis), and consequently this $S$. sonnei strain can synthesize D,D-heptose, but cannot synthesize the L,D-heptose. In the cases of the $S$. sonnei $R 41$ and 4303 strains the heptose constituents are L-glycero-Dmannoheptoses (L,D-heptose).
The presence of Kdo-s could not be detected in the hydrolyzed core oligosaccharides by TLC, GC-MS and CE-LIF. The explanation is that during mild acid hydrolysis of endotoxins (see Experimental) the carbohydrate part separates from the lipid A by the cleavage of the glycosidic bond of the Kdo, the reducing terminal residue of the core oligosaccharide in each endotoxin. In this way, lipid A moiety of endotoxins remains complete, but the core part is truncated by loosing the Kdo constituents due to various alterations and degradation reactions (besides the cleavage of the ketosidic bond). ${ }^{26-28}$

\section{CONCLUSION}

Shigella sonnei rough mutants $4350,562 H, R 41$ and 4303 are called isogenic mutants, as they are generated from the same "parent mutant" strain, $S$. sonnei 4303 (phase II). This is confirmed now by this study, in which the chemical compositions of the endotoxins extracted from the Gram-negative bacteria were determined by TLC, GC-MS and CE-LIF experiments.

The identification of the carbohydrates in the evaluation of the GC-MS experiments was accomplished by the help of the MS library, and by comparing retention times with that of the standards, as well as comparing relative retention times, which helped to assign the correct configuration of the sugar units (to differentiate between e.g. galactose and glucose or between the different heptoses).

Several studies on the structure of the S. sonnei core region have been performed previously, ${ }^{11,12,14}$ but none of them used capillary electrophoretic methods. The resolution of the monosaccharide components in the TLC experiments was not as high as in the CE-LIF runs. CE-LIF provided a higher resolution of sugar components and made the differentiation of the heptose components possible. Although, the carbohydrate labeling with APTS have shown difficulties previously, ${ }^{24}$ the qualitative analysis of the samples by CE-LIF resulted in clearly interpretable data, since the applied dye-excess increased only the peak of the dye, which avoided the competition among the sample molecules (monosaccharides).

Based on the results, we can conclude that the CE-LIF experiments together with the GC-MS analyses are suitable methods to discover the carbohydrate composition of polysaccharides, such as bacterial lipopolysaccharides. Further MALDI-TOF studies had to be performed, however, to clarify the core and lipid A composition and also to structural variability (e.g. phosphorylation) of these $R$-type endotoxins. ${ }^{16}$

Acknowledgements. The work was supported by the grants GVOP-3.2.1-0168, CEEPUS HU-0010, RET 008/2005 and OTKA-NKTH-NI-68863. Á.D. acknowledges the support of the János Bolyai Research Scholarship (Hungarian Academy of Sciences). 


\section{REFERENCES}

1. M. Lukacova, I. Barak, and J. Kazar, Clin. Microbiol. Infect. 14 (2008) 200-206.

2. M. Caroff and D. Karibian, Carbohydr. Res. 338 (2003) 2431-2447.

3. K. Rauss, I. Kétyi, A. Vertényi, and S. Vörös, Acta Microbiol. Acad. Sci. Hung. 8 (1961) 53-63.

4. C. R. H. Raetz and C. Whitfield, Annu. Rev. Biochem. 71 (2002) 635-700.

5. D. Kopecko, O. Washington, and S. Formal, Infect. Immun. 29 (1980) 207-214.

6. A. T. Maurelli and P. J. Sansonetti, Annu. Rev. Microbiol. 42 (1988) 127-150.

7. T. Kontrohr and B. Kocsis, J. Biol. Chem. 256 (1981) 7715-7718.

8. B. Kocsis and T. Kontrohr, J. Biol. Chem. 259 (1984) $11858-$ 11860 .

9. B. Kocsis, T. Kontrohr, V. László, and H. Milch, Acta Microbiol. Acad. Sci. Hung. 27 (1980) 217.

10. T. Kontrohr and B. Kocsis, J. Chromatogr. 354 (1986) 417-423.

11. T. Kontrohr and B. Kocsis, Eur. J. Biochem. 88 (1978) 267-273.

12. E. Romanowska and M. Mulczyk, Eur. J. Biochem. 5 (1968) 109-113.

13. E. Romanowska and V. Reinhold, Eur. J. Biochem. 36 (1973) $160-166$.

14. A. Gamian and E. Romanowska, Eur. J. Biochem. 129 (1982) 105-109.

15. B. Serény, Acta Microbiol. Acad. Sci. Hung. 8 (1961) 159-172.

16. A. Kilár, A. Dörnyei, A. Bui, Z. Szabó, B. Kocsis, and F. Kilár,
J. Mass Spectrom. 46 (2011) 61-70.

17. M. J. Osborn, Isolation of Phage-resistant Mutants of Salmonella typhimurium, in: E. F. Neufeld and V. Ginsburg (Eds.), Methods in Enzymology, Complex Carbohydrates, Vol. 8, Academic Press, New York, 1966, pp. 152-161.

18. C. Galanos, O. Lüderitz, and O. Westphal, Eur. J. Biochem. 9 (1969) 245-249.

19. E. Vinogradov, Carbohydr. Res. 337 (2002) 961-963.

20. M. Dubois, K. Gillis, J. Hamilton, P. Rebers, and F. Smith, Anal. Chem. 28 (1956) 350-356.

21. E. Stahl, Dünnschichtchromatographie, Springer, Berlin, 1962.

22. W. E. Trevelyan, D. P. Procter, and J. S. Harrison, Nature 166 (1950) 444-445.

23. J. Sawardeker, J. Sloneker, and A. Jeanes, Anal. Chem. 37 (1965) 1602-1604.

24. A. Bui, B. Kocsis, and F. Kilár, J. Biochem. Biophys. Meth. 70 (2008) 1313-1316.

25. H. Guzlek, A. Graziani, and P. Kosma, Carbohydr. Res. 340 (2005) 2808-2811.

26. R. Chaby, D. Charon, M. Caroff, S. R. Sarfati, and F. Trigalo, Estimation of 3-Deoxy-D-manno-2-octulosonic acid in Lipopolysaccharides: An Unsolved Problem, in: R. L. Whistler, J. N. BeMiller, and D. H. Shaw (Eds.), Methods in Carbohydrate Chemistry, Lipopolysaccharides. Separation and analysis. Gycosylated polymers, Vol. 9, E-Publishing, New York, 1993, p. 33.

27. S. Wilkinson, Prog. Lipid Res. 35 (1996) 283-343.

28. M. M. A. Olsthoorn, J. Haverkamp, and J. E. Thomas-Oates, J. Mass Spectrom. 34 (1999) 622-636. 